Anaesthesist 2022 $\cdot 71: 599-607$

https://doi.org/10.1007/s00101-022-01100-3

Eingegangen: 8. November 2021

Überarbeitet: 1. Januar 2022

Angenommen: 13. Februar 2022

Online publiziert: 7. März 2022

(c) Der/die Autor(en) 2022

\section{„Resuscitative endovascular balloon occlusion of the aorta" bei schwer verletzten Patienten im Schockraum: eine Fallserie}

\author{
Jürgen Knapp ${ }^{1,2,5} \cdot$ Dominik A. Jakob ${ }^{3} \cdot$ Tobias Haltmeier $^{4} \cdot$ Beat Lehmann ${ }^{3}$. \\ Wolf E. Hautz ${ }^{3}$ \\ 'Klinik für Anästhesiologie und Schmerztherapie, Inselspital, Universitätsspital Bern, Universität Bern, \\ Bern, Schweiz \\ ${ }^{2}$ Klinik für Anästhesie, Intensivmedizin und Schmerztherapie, Spital Schwyz, Schwyz, Schweiz \\ ${ }^{3}$ Universitäres Notfallzentrum, Inselspital, Universitätsspital Bern, Universität Bern, Bern, Schweiz \\ ${ }^{4}$ Klinik für Viszerale Chirurgie und Medizin, Inselspital, Universitätsspital Bern, Universität Bern, Bern, \\ Schweiz \\ ${ }^{5}$ Klinik für Anästhesiologie und Schmerztherapie, Universitätsspital Bern, Universität Bern, Bern, Schweiz
}

Zusammenfassung

Eine Hämorrhagie ist in 30-40\% der Patienten, die im Rahmen eines Traumas versterben, todesursächlich und die häufigste vermeidbare Todesursache. Für nichtkomprimierbare Blutungen im Bereich des Abdomens oder des Beckens wird in den letzten Jahren zunehmend (wieder) die Anwendung der "resuscitative endovascular balloon occlusion of the aorta" (REBOA) zur temporären Blutungskontrolle diskutiert. Seit August 2020 steht im Schockraum des Universitären Notfallzentrums des Universitätsspital Bern die REBOA als Therapieoption im Rahmen der Schwerverletztenversorgung zur Verfügung. Wir berichten in dieser Fallserie unsere Erfahrungen aus allen 7 Anwendungen im Laufe des ersten Jahres.

\title{
Schlüsselwörter
}

REBOA · Endovaskuläre Therapie · Schwerstverletztenversorgung · Schock · Behandlungsmethoden

\section{Einleitung}

Bei Schwerverletzen ist die Hämorrhagie verantwortlich für 30-40\% der Todesfälle und gleichzeitig die häufigste potenziell vermeidbare Todesursache [1-5]. Die überwiegende Mehrzahl der lebensbedrohlichen Blutungen in der zivilen Notfallmedizin in Europa findet sich in Becken und Abdomen [1, 6, 7]. Der Einsatz der „resuscitative endovascular balloon occlusion of the aorta" (REBOA) zur temporären Blutungskontrolle bei diesen Verletzungen hat in den letzten Jahren ein gewisses „Revival“ erfahren. Nachdem die erste Beschreibungen einer aortalen Ballontamponade zur Versorgung einer intraabdominellen Verletzung aus der Militärmedizin zu Zeiten des Koreakrieges stammen $[8,9]$, fand in den vergangenen Jahrzehnten die Technik im Wesentlichen in einigen gefäßchirurgischen Zentren zur temporären Blutungskontrolle beim rupturiertem Bauchaortenaneurysma Anwendung [10, 11]. Durch die technische Weiterentwicklung des Materials und den Rückgang der verfahrensbedingten Komplikationen wird die REBOA inzwischen wieder v.a. im Rahmen von Beckenfrakturen zur temporären Blutungskontrolle als vielversprechende Methode diskutiert, um die Zeit bis zur operativen oder zur interventionell-angiologischen Blutstillung zu überbrücken [12-15]. In der aktuellen S3-Leitlinie "Polytrauma/ Schwerverletztenversorgung" wird die REBOA im Sinne einer Expertenmeinung (Empfehlungsgrad 0) bei „kreislaufinsta- 
Tab. 1 Übersicht über aktuelle Leitlinien und Empfehlungen zum Einsatz der "resuscitative endovascular balloon occlusion of the aorta“ (REBOA)

\section{Publikation}

S3-Leitlinie Polytrauma/

Schwerverletztenversorgung

(Deutschland) [16]

S3-Leitlinie zu Screening, Diagnostik, Therapie und Nachsorge des Bauchaortenaneurysmas

(Deutschland) [17]

The European guideline on management of major bleeding and coagulopathy following trauma: fifth edition (Europa) [18]

Consensus on resuscitative endovascular balloon occlusion of the aorta: A first consensus paper using a Delphi method (internationale Konsenspublikation) [19]

Empfehlungen

„Die temporäre endovaskuläre Ballonokklusion der Aorta (REBOA) oder anderer großer Gefäße kann bei kreislaufinstabilen Patienten in extremis zur Wiederherstellung einer zentralen Zirkulation angewandt werden, um das Zeitfenster bis zur definitiven operativen oder interventionellen Therapie zu vergrößern." (Empfehlungsgrad 0)

„Der Einsatz des aortalen Okklusionsballons bei EVAR sollte bei Patienten mit hypovolämischem Schock erwogen werden."

(Evidenzgrad 4/Empfehlungsgrad B, starker Konsens)

"We suggest that the use of aortic balloon occlusion be considered only under extreme circumstances in patients with pelvic fracture in order to gain time until appropriate bleeding control measures can be implemented."

(Grade 2C)

"The expert panel reached consensus that REBOA can be used in austere military setting, emergency departments, operating rooms and intensive care units, but disagrees with the statement that REBOA is feasible in the prehospital setting (20/36, 55.6\%).

Panel members reached consensus that REBOA is indicated in the following patient populations: traumatic abdominopelvic hemorrhage, hemorrhage arising from a ruptured aneurysm, patients with severe postpartum hemorrhage (PPH) [...].

The physiological parameters to select patients for REBOA use are trauma victims [...] with an initial systolic blood pressure of $<90 \mathrm{~mm} \mathrm{Hg}$ who do not respond at all to initial fluid or blood products and trauma victims with an ATLS class IV hypovolemic shock. Patients in extremis (no pulse, no blood pressure) should not be considered for REBOA with $25 / 36 ; 69.4 \%$ of panel members agreeing. [...] The panel did not support the statement that trauma victims with a systolic pressure $>90 \mathrm{~mm} \mathrm{Hg}$, but with a mechanism of injury suspicious for high early bleeding risk (severe pelvic fracture, positive FAST exam) should be eligible for REBOA (7/36; 19.4\%)."

Joint statement from the American College of Surgeons Committee on Trauma (ACS COT) and the American College of Emergency Physicians (ACEP) regarding the clinical use of Resuscitative Endovascular Balloon Occlusion of the Aorta (REBOA)

(USA) [20]

Resuscitative endovascular balloon occlusion of the aorta in NSW trauma (Australien) [21]

Clinical use of resuscitative endovascular balloon occlusion of the aorta (REBOA) in civilian trauma systems in the USA, 2019: a joint statement from the American College of Surgeons Committee on Trauma, the American College of Emergency Physicians, the National Association of Emergency Medical Services Physicians and the National Association of Emergency Medical Technicians

(USA) [22]

Resuscitative endovascular balloon occlusion of the aorta (REBOA) in patients with major trauma and uncontrolled haemorrhagic shock: a systematic review with meta-analysis (Italien, Metaanalyse im Rahmen der Leitlinien-Entwicklung zur Schwerverletztenversorgung für das Istituto Superiore di Sanità) [23]

ATLS advanced trauma life support, EVAR endovascular aortic repair, FAST focused assessment with sonography in trauma, NSW New South Wales, TIC Trauma Innovation Committee
"REBOA is indicated for traumatic life-threatening hemorrhage below the diaphragm in patients in hemorrhagic shock who are unresponsive or transiently responsive to resuscitation."

"REBOA is indicated for patients arriving in arrest from injury due to presumed life-threatening hemorrhage below the diaphragm. No evidence exists for the recommended duration of arrest and use of REBOA but should be used within the same time period as would resuscitative thoracotomy."

"Currently, there is insufficient high-level evidence on REBOA's effectiveness for improving mortality outcomes in trauma. [...] Uncertainty around which patient cohort is likely to most benefit (i.e. survival benefit) from REBOA remains a fundamental question; identifying the optimal patient group, where and when REBOA is implemented are some crucial questions, when answered, may provide greater clarity. TIC recommends further prospective studies are necessary to understand the role of REBOA in torso haemorrhage in trauma and which patient group is likely to benefit from REBOA intervention."

"There is no high-grade evidence demonstrating that REBOA improves outcomes or survival compared with standard treatment of severe traumatic hemorrhage. [...] At a small number of high-volume trauma centers experienced with this procedure, REBOA has emerged as a protocolized option in select patients with non-compressible torso trauma. [...] REBOA is a tool that should only be employed as part of a larger system of damage control resuscitation, definitive hemorrhage control, and postoperative critical care. It is used to temporize patients at high risk of mortality from non-compressible torso hemorrhage."

"Our findings on overall mortality suggest a positive effect of REBOA among non-compressible torso injuries when compared to RT but no differences compared to no-REBOA. Variability in indications and patient characteristics prevents any conclusion deserving further investigation. REBOA should be promoted in specific training programs in an experimental setting in order to test its effectiveness and a randomized trial should be planned." 


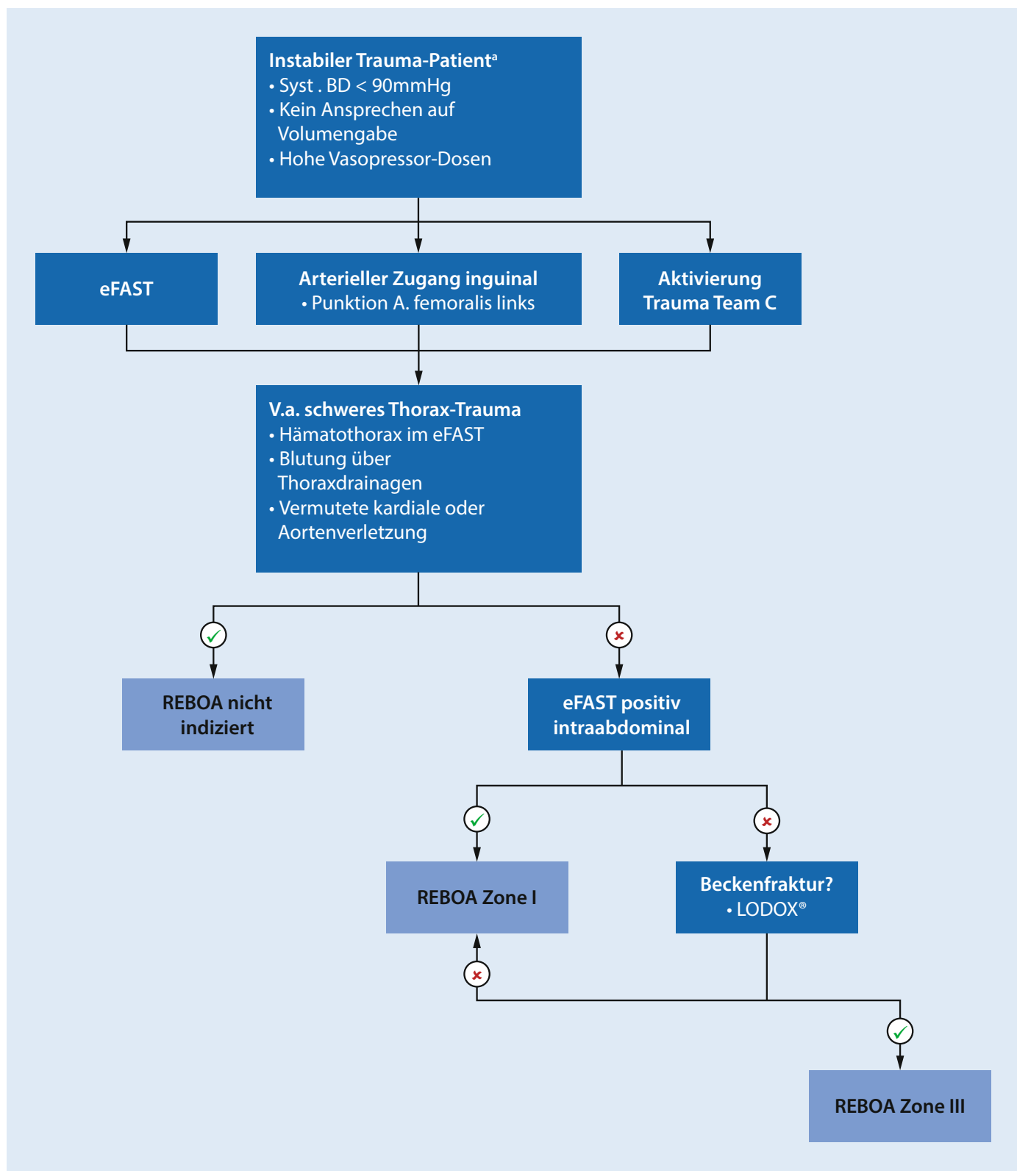

Abb. $1 \triangleleft$ Algorithmus zur Anwendung der "resuscitative endovascular balloon occlusion of the aorta" (REBOA) des Universitären Notfallzentrums des Universitätsspitals Bern. ${ }^{a}$ Traumatic cardiac arrest im Schockraum oder $<10 \mathrm{~min}$ vorEintreffen:Notfallthorakotomie erwägen. $B D$ Blutdrucke, eFAST extended focused assessment using sonography in trauma, $R E-$ $B O A$ resuscitative endovascular balloon occlusion of the aorta, V.a. Verdacht auf

bilen Patienten in extremis" empfohlen [16]. Eine Übersicht über die aktuellen internationalen Leitlinien und leitliniennahen Empfehlungen zum Einsatz der REBOA bietet - Tab. 1.

Wir haben im Universitären Notfallzentrum des Universitätsspitals Bern seit 2020 einen Algorithmus zur Durchführung einer REBOA bei hämodynamisch instabilen Traumapatienten etabliert ( $\bullet$ Abb. 1). Seither wurde das REBOA-Manöver über den Zeitraum von 12 Monaten bei 7 Patienten durchgeführt. Im Folgenden möchten wir konsekutiv alle Fälle kurz berichten und unsere Erfahrungen zur Diskussion stellen. Die in den einzelnen Fallberichten ge- schilderten Verletzungen sind die Diagnosen, die nach Abschluss der kompletten Diagnostik (inklusive Computertomographie) im Rahmen der Schockraumversorgung gestellt wurden. Die Entscheidung zur REBOA musste in den meisten Fällen aber sehr frühzeitig und meist nur anhand der Informationen zur Unfallkinematik, der Vitalparameter und der Erstuntersuchung (in der Regel inklusive fokussierter Sonographie [„extended focused assessment with sonography for trauma", eFAST] und evtl. Röntgenaufnahme des Thorax und Beckens) im Schockraum gestellt werden.

\section{Fallberichte}

Fall 1

Unfallmechanismus. Der Patient wurde bei Baumfällarbeiten zwischen 2 Bäumen eingeklemmt.

Initiale Arbeitsdiagnose. Beckentrauma, stumpfes Thorax- und Abdominaltrauma.

Hämodynamische Situation. Bei Aufnahme in den Schockraum hämodynamisch instabil. Mittlerer arterieller Druck (MAD) $50-60 \mathrm{~mm} \mathrm{Hg}$ unter Noradrenalin bis $0,1 \mu \mathrm{g} / \mathrm{kgKG}$ und $\mathrm{min}$. 


\begin{tabular}{|c|c|c|c|c|c|c|c|c|}
\hline $\begin{array}{l}\text { Fall, Ge- } \\
\text { schlecht, } \\
\text { Alter in } \\
\text { Jahren }\end{array}$ & $\begin{array}{l}\text { Führende } \\
\text { Verletzung }\end{array}$ & $\begin{array}{l}\text { Zeit } \\
\text { bis Ab- } \\
\text { schluss } \\
\text { des } \\
\text { eFAST }\end{array}$ & Zeit im Schockraum bis REBOA & $\begin{array}{l}\text { Zeit } \\
\text { bis Ab- } \\
\text { schluss } \\
\text { CT-Dia- } \\
\text { gnostik }\end{array}$ & $\begin{array}{l}\text { Gesamtzeit } \\
\text { der Schock- } \\
\text { raumver- } \\
\text { sorgung }\end{array}$ & $\begin{array}{l}24- \\
h- \\
\text { Über- } \\
\text { le- } \\
\text { ben }\end{array}$ & $\begin{array}{l}\text { Kranken- } \\
\text { hausent- } \\
\text { lassung }\end{array}$ & Todesursache \\
\hline $\begin{array}{l}\text { Fall 1, m, } \\
68\end{array}$ & $\begin{array}{l}\text { Schweres Be- } \\
\text { ckentrauma }\end{array}$ & $3 \min$ & $32 \mathrm{~min}$ & $50 \mathrm{~min}$ & $\begin{array}{l}160 \text { min } \\
\text { (inkl. Be- } \\
\text { ckenzwin- } \\
\text { ge) }\end{array}$ & Ja & Nein & $\begin{array}{l}\text { Septisches Multior- } \\
\text { ganversagen }\end{array}$ \\
\hline $\begin{array}{l}\text { Fall 2,w, } \\
58\end{array}$ & $\begin{array}{l}\text { Schweres Schä- } \\
\text { del-Hirn-Trau- } \\
\text { ma, Wirbel- } \\
\text { säulentrauma }\end{array}$ & $15 \mathrm{~min}$ & $15 \mathrm{~min}$ & $67 \mathrm{~min}$ & $\begin{array}{l}225 \text { min } \\
\text { (inkl. exter- } \\
\text { ne Ventri- } \\
\text { keldraina- } \\
\text { ge) }\end{array}$ & $\mathrm{Ja}$ & Nein & $\begin{array}{l}\text { Schweres Schädel- } \\
\text { Hirn-Trauma }\end{array}$ \\
\hline $\begin{array}{l}\text { Fall 3, m, } \\
33\end{array}$ & $\begin{array}{l}\text { Schweres Be- } \\
\text { ckentrauma, } \\
\text { beidseitige } \\
\text { Unterschen- } \\
\text { kelamputation }\end{array}$ & $6 \mathrm{~min}$ & $21 \mathrm{~min}$ & $45 \mathrm{~min}$ & $126 \min$ & $\mathrm{Ja}$ & Nein & Maligner Mediainfarkt \\
\hline $\begin{array}{l}\text { Fall 4, m, } \\
40\end{array}$ & $\begin{array}{l}\text { Schweres Be- } \\
\text { ckentrauma }\end{array}$ & $2 \min$ & $40 \mathrm{~min}$ & $78 \mathrm{~min}$ & $\begin{array}{l}176 \text { min } \\
\text { (inkl. Be- } \\
\text { ckenzwin- } \\
\text { ge) }\end{array}$ & $\mathrm{Ja}$ & $\mathrm{Ja}$ & - \\
\hline $\begin{array}{l}\text { Fall 5, m, } \\
62\end{array}$ & $\begin{array}{l}\text { Schweres Ab- } \\
\text { dominal- und } \\
\text { Beckentrauma }\end{array}$ & $5 \mathrm{~min}$ & $--^{a}$ & $76 \mathrm{~min}$ & $\begin{array}{l}262 \text { min } \\
\text { (Wartezeit } \\
\text { auf OP) }\end{array}$ & $\mathrm{Ja}$ & $\mathrm{Ja}$ & - \\
\hline $\begin{array}{l}\text { Fall 6, m, } \\
64\end{array}$ & $\begin{array}{l}\text { Traumatischer } \\
\text { Herz-Kreislauf- } \\
\text { Stillstand }\end{array}$ & $13 \mathrm{~min}$ & $\begin{array}{l}\text { Sofortige linksseitige Thora- } \\
\text { kotomie, Klemmung der Aorta } \\
\text { thoracica und Bülau-Drainage } \\
\text { rechtsseitig, Umstellung auf } \\
\text { REBOA nach } 12 \text { min }\end{array}$ & $94 \mathrm{~min}$ & $99 \min$ & Nein & - & $\begin{array}{l}\text { Nichtbeherrschbare } \\
\text { abdominelle und tho- } \\
\text { rakale Blutung nach } \\
\text { traumatischem Herz- } \\
\text { Kreislauf-Stillstand }\end{array}$ \\
\hline $\begin{array}{l}\text { Fall 7, m, } \\
76\end{array}$ & $\begin{array}{l}\text { Traumatischer } \\
\text { Herz-Kreislauf- } \\
\text { Stillstand }\end{array}$ & $3 \min$ & $7 \mathrm{~min}$ & $36 \mathrm{~min}$ & $\begin{array}{l}98 \mathrm{~min} \\
\text { (Überlei- } \\
\text { tung in } \\
\text { palliative } \\
\text { Therapie) }\end{array}$ & Nein & - & $\begin{array}{l}\text { Schweres diffuses } \\
\text { Hirnödem nach } \\
\text { prähospitalem trau- } \\
\text { matischem Herz- } \\
\text { Kreislauf-Stillstand bei } \\
\text { Polytrauma }\end{array}$ \\
\hline \multicolumn{9}{|c|}{$\begin{array}{l}m \text { männlich, } w \text { weiblich, eFAST "extended focused assessment with sonography for trauma", CT Computertomographie, REBOA resuscitative endovascular } \\
\text { balloon occlusion of the aorta } \\
\text { 'Die Entscheidung zur REBOA fiel in diesem Fall erst, als es nach Abschluss der Schockraumdiagnostik bei der Eröffnung des Beckengurts zur Vorbereitung für } \\
\text { die operative Versorgung zur hämodynamischen Instabilität kam }\end{array}$} \\
\hline
\end{tabular}

REBOA-Manöver. Problemlose, ultraschallgestützte Einlage einer 7-F-Schleuse femoral-arteriell rechts und konsekutives Einbringen einer ER-REBOA ${ }^{\circledR}$ (Fa. Prytime Medical, Texas, USA) in Zone III innerhalb von 9 min (gemessen von der Entscheidung für das REBOA-Manöver bis zur Ballonokklusion der Aorta). Lagekontrolle durch Ableiten arterieller Druckkurven an Schleuse und Katheterspitze. Nach REBOA MAD $70-80 \mathrm{~mm} \mathrm{Hg}$, kein Noradrenalinbedarf mehr.

Umstellung auf partielle REBOA (d.h. nur noch partiell geblockter Ballon, sodass in der invasiv gemessenen Blutdruckkurve distal der Aortenokklusion wieder eine leichte Pulsation zu erkennen ist) und vollständiger Rückzug des Katheters innerhalb der folgenden 45 min unter massiver Volumensubstitution (Kristalloide, Erythrozytenkonzentrate und Frischplasma) und nach Stabilisierung und Anlegen einer Beckenzwinge schließlich Verlegung in die Angiographie-Suite. Die einliegende femoral-arterielle Schleuse konnte für die Embolisation der pelvinen Blutungsquelle genutzt werden.

Wesentliche Verletzungen. Beckenfraktur (C-Verletzung) mit bilateraler Sakrumfraktur sowie Schambeinastfraktur rechts und Sprengung des Iliosakralgelenks, Rippenserienfraktur rechts mit Pneumotho- rax, große Morel-Lavallée-Verletzung gluteal rechtsseitig, Milzlazeration $I^{\circ}$.

Behandlungsergebnis. Exitus letalis 22 Tage nach dem Trauma nach Entscheid für eine palliative Therapie bei septischem Multiorganversagen bei im Verlauf diagnostizierter Rektum- und Dünndarmperforation im Rahmen eines Abrisses des Mesenteriums sowie multiplen ischämischen Grenzzoneninfarkten im Mediastromgebiet bei zerebrovaskulärer Verschlusskrankheit. 


\section{Fall 2}

Unfallmechanismus. Sturz aus dem 3. Stock.

Initiale Arbeitsdiagnose. Schweres Polytrauma mit führendem Schädel-Hirn-Trauma, eFAST: wenige freie Flüssigkeit im Morison-Pouch.

Hämodynamische Situation. Hämodynamisch instabil, MAD 30-45 mm Hg unter $0,08 \mu \mathrm{g} / \mathrm{kgKG}$ und min Noradrenalin, Herzfrequenz (HF) 130/min.

REBOA-Manöver. Problemlose, ultraschallgestützte Einlage einer 7-F-Schleuse femoral-arteriell rechts und konsekutives Einbringen einer ER-REBOA innerhalb von $13 \mathrm{~min}$. Entgegen den klinikinternen Vorgaben (•Abb. 1) wird der Ballon trotz positivem eFAST in Zone III, nicht in Zone I platziert. Lagekontrolle durch Ableiten arterieller Druckkurven an Schleuse und Katheterspitze. Umstellung auf partielle REBOA unter kontinuierlicher Volumengabe mit kristalloiden Infusionslösungen und Blutprodukten und vollständige Entfernung des Katheters nach $90 \mathrm{~min}$.

Nach REBOA: MAD 80-100 mm Hg unter $0,02-0,04 \mu \mathrm{g} / \mathrm{kgKG}$ und min Noradrenalin, HF 100-110/min.

Wesentliche Verletzungen Traumatische Subarachnoidalblutung, Dissektion $\operatorname{der} A$. vertebralis links im $V_{2}$-Segment, instabile Wirbelkörperfraktur Halswirbelkörper (HWK) 3/4, Lungenlazeration links mit kleinem Pneumothorax.

Behandlungsergebnis. Exitus letalis 33 Tage nach dem Trauma nach Entscheid für eine palliative Therapie bei fehlender Aufwachreaktion nach Ausschluss reversibler Ursachen und irreversibler hoher Schädigung des Rückenmarks in Höhe HWK 3/4.

Fall 3

Unfallmechanismus. Patient wurde von einem Zug erfasst.

Initiale Arbeitsdiagnose. Beckentrauma, Thoraxtrauma, beidseitige Unterschenkel- amputation, eFAST: Pneumothorax links, keine freie Flüssigkeit im Abdomen.

Hämodynamische Situation. Hämodynamisch instabil, MAD $70 \mathrm{~mm} \mathrm{Hg}$ unter $0,25 \mu \mathrm{g} / \mathrm{kgKG}$ und min Noradrenalin, Herzfrequenz 140/min.

REBOA-Manöver. Beim wachen Patienten frustraneSchleuseneinlage unter Ultraschallkontrolle in der rechten Femoralarterie (Draht nicht zu positionieren). Schließlich problemlose, ultraschallgestützte Einlage einer 7-F-Schleuse femoral-arteriell links und konsekutives Einbringen eines ER-REBOA ${ }^{\circledR}$-Katheters innerhalb von 7 min in Zone III. Aortenokklusion zeitgleich mit Einleitung der Intubationsnarkose. Lagekontrolle durch Ableiten arterieller Druckkurven an Schleuse und Katheterspitze. Umstellung auf partielle REBOA unter kontinuierlicher Volumengabe und Übernahme des Patienten mit partiell geblocktem Katheter in den OP.

Nach REBOA: MAD 80-100 mm Hg ohne Katecholamine, HF 80-100/min.

Wesentliche Verletzungen. Rippenserienfraktur links, kleiner Hämatopneumothorax links, Sternumfraktur, Acetabulumfraktur rechts, beidseitige Hüftluxation, beidseitige komplette Unterschenkelamputation, blutend trotz straff anliegender Tourniquets.

Behandlungsergebnis. Exitus letalis 18 Tage nach dem Trauma nach Entscheid zur palliativen Therapie bei malignem Mediainfarkt unklarer Ursache.

Fall 4

Unfallmechanismus. Fahrradfahrer von Lkw überrollt.

Initiale Arbeitsdiagnose. Beckenfraktur, ausgedehnte Weichteilverletzungen, eFAST: negativ.

Hämodynamische Situation. Hämodynamisch instabil, MAD $70-80 \mathrm{~mm} \mathrm{Hg}$ unter Noradrenalin auf 0,02-0,05 $\mu \mathrm{g} /$ kgKG und min und Massivtransfusion, HF 90-130/min.
REBOA-Manöver. Bei initial mäßiger Volumenresponse Einlage einer 7-F-Schleuse femoral-arteriell links unter Sicht (aufgrund der ausgedehnten DécollementVerletzung) und beim wachen Patienten problemlos. Anlage einer Beckenzwinge im Schockraum nach Intubationsnarkose. Im Verlauf bei zunehmender hämodynamischer Instabilität Einbringen eines ER$\mathrm{REBOA}^{\circledR}$-Katheters über die Schleuse in Zone II (Ziel war Zone III) und Verlegung des Patienten in den OP.

Nach REBOA: MAD $60-80 \mathrm{~mm} \mathrm{Hg}$ ohne Katecholamine, HF 60-70/min.

Wesentliche Verletzungen. Décollement von $35-40 \%$ der Körperoberfläche an Rumpf und Oberschenkel beidseits, Beckenfraktur (C-Verletzung) mit spinopelviner Dissoziation, Trümmerfraktur des Sakrums, bilaterale Schambeinasttrümmerfraktur, Impressionsfraktur des rechten Acetabulums mit zentraler Luxation des Hüftkopfes, Dünndarmperforation, Harnblasenruptur.

Behandlungsergebnis. Verlegung in ein Verbrennungszentrum aufgrund der ausgedehnten Weichteilverletzungen am 13. Tag nach dem Trauma, wach, orientiert, unter suffizienter Analgesie.

Fall 5

Unfallmechanismus. Motorradunfall.

Initiale Arbeitsdiagnose. Thoraxtrauma, Abdominaltrauma, Beckentrauma, eFAST: Hämatopneumothorax rechts, freie Flüssigkeit im Abdomen.

Hämodynamische Situation. Hämodynamisch instabil, MAD 60-70 mm Hg unter Noradrenalin $0,07 \mu \mathrm{g} / \mathrm{kgKG}$ und min, HF $100-110 / \mathrm{min}$.

REBOA-Manöver. Bei Eröffnung des Beckengurts in Vorbereitung zur operativen Stabilisierung nach Abschluss der Diagnostikkam es zur massiven hämodynamischen Instabilität, sodass wir uns zur sofortigen Anlage eines REBOA-Katheters entschieden. Einlage der Schleuse problemlos unter Ultraschallkontrolle und Positionierung des Ballons in Zone I. 
Nach REBOA: MAD 60-70 mm Hg unter rückläufiger Noradrenalindosierung bis auf $0,01 \mu \mathrm{g} / \mathrm{kgKG}$ und $\mathrm{min}, \mathrm{HF} 70-80 / \mathrm{min}$. Übernahme in den OP zur operativen Stabilisierung des Beckens.

Wesentliche Verletzungen. Contusio cordis, kleiner Hämatopneumothorax rechts, dislozierte Rippenserienfraktur rechts, Milzlazeration und Leberlazeration $\mathrm{II}^{\circ}$, aktive intraabdominale Blutung bei Lazeration A. hepatica und Mesoeinriss, komplexe Beckenfraktur (C-Verletzung) mit aktiver Blutung, Blasenruptur.

Behandlungsergebnis. Entlassung am 68. Tag nach dem Trauma in die Rehabilitation in gutem Allgemeinzustand.

\section{Fall 6}

Unfallmechanismus. Der Patient wurde von einem Lkw überrollt. Kurz nach Eintreffen des Rettungsdienstes wird der Patient reanimationspflichtig bei pulsloser elektrischer Aktivität des Herzens (PEA).

Initiale Arbeitsdiagnose. Traumatischer Herz-Kreislauf-Stillstand.

Hämodynamische Situation und REBOA-Manöver. Bei Aufnahme in den Schockraum war der Patient nach wie vor reanimationspflichtig. Daher entschlossen wir uns zur sofortigen linksseitigen Thorakotomie und Klemmung der Aorta (Zeit von Ankunft im Schockraum bis Klemmen der Aorta $4 \mathrm{~min}$ ), parallel wurde rechtsseitig eine Thoraxdrainage in BülauPosition eingelegt. Unter zusätzlicher Massivtransfusion konnte so innerhalb von 5 min eine Wiederherstellung eines Spontankreislaufs („restoration of spontaneous circulation", ROSC) erzielt werden. Im anschließend durchgeführten eFAST fand sich abdominell keine freie Flüssigkeit, sodass während der Vorbereitungen zur Anlage einer Beckenzwinge eine femoralarterielle Schleuse (mittels "Cut-down"Technik) und hierüber eine REBOA in Zone III platziert wurden. Dieses Manöver konnte innerhalb von $5 \mathrm{~min}$ und ohne erneute hämodynamische Instabilität durchgeführt werden.
Wesentliche Verletzungen. Die Aufnahme in den Schockraum erfolgt unter kardiopulmonaler Reanimation bei pulsloser elektrischer Aktivität des Herzens (PEA). „Open book"-Verletzung des Beckens, Rippenserienfrakturen beidseits.

Behandlungsergebnis. Im Laufe der operativen Versorgung des Patienten kam es trotz Massivtransfusion und intensiver Substitution von Gerinnungsfaktoren zur diffusen Blutungstendenz in Abdomen und Thorax, die auch chirurgisch nicht weiter zu kontrollieren war. Daher wurde interdisziplinär die Entscheidung zum Therapieabbruch getroffen. Exitus letalis $5 \mathrm{~h}$ nach Aufnahme.

\section{Fall 7}

Unfallmechanismus. Gleitschirmabsturz aus ca. $30 \mathrm{~m}$ Höhe. Kurz nach Eintreffen des Rettungshubschraubers wird der Patient während der Versorgung reanimationspflichtig, ROSC prähospital nach einer Reanimationsdauer von $20 \mathrm{~min}$.

Initiale Arbeitsdiagnose. Traumatischer Herz-Kreislauf-Stillstand, eFAST: freie Flüssigkeit im Koller-Pouch, wenig Perikarderguss, hämodynamisch nicht relevant.

Hämodynamische Situation. Zwei Minuten nach Aufnahme des Patienten in den Schockraum kommt es erneut zu einem Kreislaufstillstand bei PEA.

REBOA-Manöver. Problemlose, ultraschallgesteuerte Einlage einer 7-F-Schleuse femoral-arteriell rechts. Unter nun notwendiger kardiopulmonaler Reanimation wird die bereits vor Eintritt des erneuten Herz-Kreislauf-Stillstandes begonnene REBOA-Einlage nun innerhalb von $2 \mathrm{~min}$ durch Einlage eines ER-REBOA ${ }^{\circledR}$-Katheters in Zone I beendet. Insgesamt dauerte die REBOA-Anlage $7 \mathrm{~min}$. Unmittelbar nach Okklusion der Aorta ROSC. Rückzug in Zone III nach Abschluss der Schockraumdiagnostik, da die Computertomographie keine relevante intraabdominelle Flüssigkeit zeigte.

Wesentliche Verletzungen. Prähospitaler traumatischer Herz-Kreislauf-Stillstand mit primär erfolgreichem ROSC, schweres
Schädel-Hirn-Trauma mit Subarachnoidalblutung, stumpfes Abdominaltrauma mit freier Flüssigkeit perisplenisch, multiple instabile Wirbelkörperfrakturen (HWK 5, Brustwirbelkörper 3, 4 und 11, Lendenwirbelkörper 1, 4 und 5), dislozierte offene Femurfraktur links.

Behandlungsergebnis. Die computertomographische Diagnostik zeigte bereits ein schweres diffuses Hirnödem, schon mit Zeichen der hypoxischen Hirnschädigung, Kornealreflexe ließen sich nicht auslösen (nach Ausschluss einer neuromuskulären Blockade), beide Pupillen waren weit und lichtstarr. Daher entschlossen wir uns noch im Schockraum zur Einleitung einer palliativen Therapie. Der Patient verstarb $5 \mathrm{~h}$ nach Aufnahme auf der Intensivstation im Beisein der Familie.

Eine Übersicht über die geschilderten Fälle bietet • Tab. 2 .

\section{Diskussion}

Die Zahl der Patienten, bei denen wir uns für die Durchführung eines REBOA-Manövers entschieden, überstieg mit 7 innerhalb eines Jahres deutlich unsere Erwartungen. Diese Zahl lag auch deutlich über den Abschätzungen aus dem Traumaregister der Deutschen Gesellschaft für Unfallchirurgie (DGU). Diese ergaben, dass in einem überregionalen Traumazentrum mindestens ein Patient/Jahr zu erwarten ist, bei dem eine REBOA indiziert ist [24]. Da das Verfahren momentan noch nur von einer kleinen Gruppe an Ärzten angewandt wird, stand die Option zur REBOA nicht täglich und nicht rund um die Uhr zur Verfügung (in der Regel nur tagsüber und werktags). Daher liegt die tatsächliche Zahl der Patienten, bei denen eine REBOA indiziert gewesen wäre, vermutlich noch höher.

Die geschilderten Fälle zeigen, dass die initiale hämodynamische Stabilisierung mittels REBOA gut gelingt. Nur einer der Patienten verstarb (nach Entscheid für eine palliative Therapie) infolge einer auch chirurgisch nicht weiter kontrollierbaren Hämorrhagie nach sowohl prähospitaler als auch innerklinisch notwendiger mechanischer Reanimation (vor REBOA). Schwerwiegende Komplikationen durch die REBOA konnten wir bei den 7 Patienten nicht beobachten. In einem Fall 
wurde der Ballon mutmaßlich beim Einführen durch die Schleuse beschädigt, und ein Aortenverschluss war nicht mehr möglich. Mit einem neuen REBOA-Katheter gelang das Manöver dann im zweiten Versuch. Bei einem Patienten konnte computertomographisch eine Dissektion der Arterienwand im Bereich der A. femoralis nachgewiesen werden, die aber differenzialdiagnostisch auch traumatisch bedingt gewesen sein könnte. Klinisch blieb diese Dissektion für den Patienten folgenlos.

Hinsichtlich der Indikationsstellung kann festgestellt werden, dass, retrospektiv betrachtet, eine REBOA bei einem Patienten (Fall 2) nicht indiziert war. Die Unfallkinematik und die hämodynamische Instabilität bei Aufnahme haben uns in diesem Fall zur Durchführung des REBOA-Manövers veranlasst, wodurch auch eine Stabilisierung des Patienten gelang. Allerdings zeigte sich in der computertomographischen Diagnostik keine Verletzung im Bereich des Beckens oder Abdomens. Die ausgeprägte Hypotonie könnte durch einen neurogenen Schock bei hoher Rückenmarkverletzung verursacht gewesen sein, das gute hämodynamische Ansprechen auf die REBOA durch die massive Erhöhung der Nachlast.

Bei reanimationspflichtigen Patienten mit möglichen thorakalen Verletzungen ist die Thorakotomie („resuscitative thoracotomy “) der REBOA vorzuziehen [13]. Daher entschlossen wir uns im Fall 6 primär zur linksseitigen Thorakotomie mit Klemmung der Aorta. Nachdem hierunter rasch ein ROSC erzielt werden konnte, die FASTUntersuchung abdominell keine freie Flüssigkeit zeigte, wechselten wir bei klinisch sicherer Beckenfraktur auf eine REBOA in der Zone III, um die thorakale Aortenklemme entfernen zu können und dadurch eine länger dauernde Ischämie der abdominellen Organe zu vermeiden.

Unsere Erfahrung aus dieser Fallserie zeigt, dass das REBOA-Manöver an sich meist innerhalb von wenigen Minuten gelingt. In einigen Fällen ging jedoch mit der Entscheidungsfindung zu diesem Manöver wertvolle Zeit verloren. Dies ist sicher z.T. darauf zurückzuführen, dass die REBOA im Rahmen der Schockraumbehandlung in unserem Notfallzentrum noch kein breit etabliertes Verfahren ist, sodass Unsicherheiten bezüglich der korrekten Indikati- on und der - insbesondere im Hinblick auf den Zeitbedarf - sinnvollen Durchführbarkeit des Manövers zu Verzögerungen führten. Mit zunehmender Erfahrung und Standardisierung der Anwendung könnten die Entscheidungsprozesse zukünftig schneller ablaufen. Insbesondere die Etablierung von „standard operating procedures" (SOP), gemeinsames Teamtraining und ein gutes Briefing bei der Vorbereitung auf die Schockraumversorgung eines schwer verletzten Patienten könnten zukünftig die Zeit bis zur Durchführung einer REBOA verkürzen. Unserer Erfahrung nach sind ein Debriefing unmittelbar nach Abschluss der Schockraumversorgung und die postakute Aufarbeitung und kritische Diskussion der Fälle beispielsweise im Rahmen eines Trauma-Board, wie dies am Inselspital Bern monatlich erfolgt, sehr hilfreich.

Als weitere Möglichkeit, den Zeitbedarf bis zum Abschluss des REBOA-Manövers zu reduzieren, diskutieren wir aktuell die standardmäßige Anlage der arteriellen Kanüle zur invasiven Blutdruckmessung in die A. femoralis bei hämodynamisch kompromittierten Patienten mit vom Unfallmechanismus her möglicher Indikation für den Einsatz der REBOA. So kann dieser Zugang jederzeit zum Einbringen der Schleuse genutzt werden, wenn die Entscheidung zur REBOA getroffen ist. Diese Idee zur Optimierung des zeitgerechten Einsatzes wird auch durch ein internationales Konsensus-Statement zur REBOA mit einer Zustimmung von $70 \%$ (28 von 40 Experten) unterstützt [19].

Essenziell bei der Einführung eines solchen Verfahrens ist auch die gute Absprache zwischen den durchführenden Fachabteilungen (in der Regel Notfallmedizin und/oder Anästhesie) und den weiteren an der Schockraumversorgung beteiligten Disziplinen, insbesondere den operativen Fächern. Im angloamerikanischen Raum wird die durch Ballonokklusion der Aorta gewonnene Zeit üblicherweise für eine unmittelbare notfallmäßige Laparotomie genutzt [25]. In allen oben beschriebenen Fällen an unserem Zentrum haben sich die beteiligten operativen Kollegen gegen eine unmittelbare Laparotomie und stattdessen für eine weiterführende Diagnostik durch Computertomographie entschieden. Bei allen Patienten konnte nach REBOA trotz initial extremer hämodynamischer Instabilität die notwendige computertomografische Diagnostik unter deutlich stabileren Bedingungen komplikationslos durchgeführt werden. In Fall 5 wurde die REBOA erst nach Abschluss der Computertomographie durchgeführt, nachdem der Patient beim Öffnen des Beckengurts instabil wurde. Die Narkoseeinleitung und weitere Vorbereitung zur operativen Versorgung verliefen darunter problemlos. Essenziell ist in diesem Zusammenhang aber festzuhalten, dass es sich bei der REBOA lediglich um eine temporäre Maßnahme zur Blutungskotrolle handelt und die weitere Versorgung (Bildgebung, Vorbereitung zur Operation oder angiologischen Intervention) unverzüglich erfolgt.

Ein paar weitere Fragen, die in Diskussionen zum Einsatz der REBOA immer wieder auftreten, können wir im Rückblick auf das eine Jahr der Anwendung in unserem Notfallzentrum beantworten:

\section{REBOA und bildgebende Diagnostik bei Polytrauma}

Hinsichtlich der computertomografischen Diagnostik muss insbesondere geklärt werden, wie mit der REBOA während der arteriellen Phase der "Traumaspirale" verfahren wird, da durch den Aortenverschluss relevante Blutungen distal davon möglicherweise nicht mehr sicher diagnostiziert werden können. Bei kompletter Deflation des Ballons für die arterielle Phase muss wieder mit schwerer hämodynamischer Instabilität gerechnet werden. Möglich ist hier eine partielle Deflation („partial REBOA“, p-REBOA). Hier besteht aber die Gefahr, dass der Ballon während der partiellen Deflation disloziert. Wir fahren daher inzwischen zunächst die arterielle Phase einer Traumaspirale mit komplett geblocktem Ballon und wiederholen diese evtl. mit nur teilweise geblocktem Ballon nochmals, falls relevante Hämatome oder unklare Extravasate von Kontrastmittel distal des Ballons festgestellt werden und es die hämodynamische Situation erlaubt. Eine gute Übersicht zu dieser Problematik bietet [26]. 


\section{Füllungsvolumen des Ballons}

Eine weitere oft diskutierte Frage insbesondere vor dem Hintergrund des variablen Durchmessers der Aorta und der Gefahr einer Verletzung der Aortenwand bei zu starker Füllung des Ballons ist die Steuerung des Füllungsvolumens bei Anwendung der REBOA. Bei gefäßchirurgischen Eingriffen wird das korrekte Füllungsvolumen fluoroskopisch kontrolliert. Dies ist im Schockraum unmöglich. Eine Alternative zur Steuerung des Ballonvolumens ist das Ableiten des invasiv gemessen Blutdrucks an der Schleuse und ein Füllen des Ballons bis zum Verschwinden der pulsatilen Blutdruckwelle. Auch diese Variante erweist sich im Geschehen eines Schockraums häufig als nicht praktikabel und zu zeitaufwändig. Daher verwenden wir für den bei uns verwendeten ER-REBOA ${ }^{\circledR}$-Katheter inzwischen einen kleinen Einwegdruckmesser (COMPASS ${ }^{\circledR}$, Fa. Centurion Medical Products, Michigan, USA), der in Reihe zwischen der Spritze und dem Konnektor zum Ballon des REBOA-Katheters geschaltet wird, und schlagen eine initiale Füllung des Ballons auf einen Druck von $160 \mathrm{~mm} \mathrm{Hg}$ vor [27].

\section{Materialvorhaltung}

Eine aktuelle Ist-Analyse zum Schockraummanagement in Deutschland ergab, dass nur $12 \%$ der Kliniken der Maximalversorgung und sogar nur $3 \%$ der Kliniken der Basisversorgung in Deutschland das Material für eine REBOA vorhalten [28]. Dies ist angesichts der Tatsache, dass die REBOA kein breit etabliertes Verfahren ist, verständlich. Für große (überregionale) Traumazentren, die regelmäßig Patienten mit schweren Beckenverletzungen versorgen, könnten eine Etablierung des Verfahrens und entsprechende Materialvorhaltung aber sinnvoll sein. Ob eine Vorhaltung in kleineren Kliniken sinnvoll ist, um entsprechende Patienten für eine Sekundärverlegung in ein überregionales Traumazentrum zu stabilisieren, lässt sich aus der aktuell vorliegenden Literatur nicht beantworten.

\section{Fazit für die Praxis}

Durch REBOA konnte bei allen 7 Patienten eine hämodynamische Stabilisierung erreicht werden, die eine computertomografische Diagnostik und ggf. Operationsvorbereitung unter relativ stabilen Bedingungen ermöglichte. Insbesondere bei der initialen Stabilisierung von hämodynamisch instabilen Patienten mit schwerem Beckentrauma sahen wir klinisch einen guten Nutzen. Schwerwiegende Komplikationen durch dieses Verfahren wurden in unserer Fallserie nicht beobachtet. Optimierungsbedarf sehen wir in der Indikationsstellung (insbesondere der Zeit bis zur Entscheidungsfindung) sowie der Ausbildung und der Aufrechterhaltung des Trainings in der Breite des Teams.

Korrespondenzadresse

PD Dr. med. Jürgen Knapp

Klinik für Anästhesiologie und Schmerztherapie, Universitätsspital Bern, Universität Bern

Freiburgstraße, 3010 Bern, Schweiz

juergen.knapp@insel.ch

Funding. Open access funding provided by University of Bern

\section{Einhaltung ethischer Richtlinien}

Interessenkonflikt. J. Knapp, D.A. Jakob, T. Haltmeier, B. Lehmann und W.E. Hautz geben an, dass kein Interessenkonflikt besteht. W.E. Hautz gibt an, Zuschüsse oder Verträge der Firmen Roche Diagnostics, MDI International, Dräger Medical und Mundipharma Research und Beratungsgelder der Firmen AO Foundation Zürich und Mundipharma Research erhalten zu haben.

Die Publikation der Fallserie erfolgt mit Zustimmung der Ethikkommission des Kantons Bern (BASEC-Nr. Req-2021-01288)

Open Access. Dieser Artikel wird unter der Creative Commons Namensnennung 4.0 International Lizenz veröffentlicht, welche die Nutzung, Vervielfältigung, Bearbeitung, Verbreitung und Wiedergabe in jeglichem Medium und Format erlaubt, sofern Sie den/die ursprünglichen Autor(en) und die Quelle ordnungsgemäß nennen, einen Link zur Creative Commons Lizenz beifügen und angeben, ob Änderungen vorgenommen wurden.

Die in diesem Artikel enthaltenen Bilder und sonstiges Drittmaterial unterliegen ebenfalls der genannten Creative Commons Lizenz, sofern sich aus der Abbildungslegende nichts anderes ergibt. Sofern das betreffende Material nicht unter der genannten Creative Commons Lizenz steht und die betreffende Handlung nicht nach gesetzlichen Vorschriften erlaubt ist, ist für die oben aufgeführten Weiterverwendungen des $\mathrm{Ma}$ terials die Einwilligung des jeweiligen Rechteinhabers einzuholen.

Weitere Details zur Lizenz entnehmen Sie bitte der Lizenzinformation auf http://creativecommons.org/ licenses/by/4.0/deed.de.

\section{Literatur}

1. Kauvar DS, Lefering R, Wade CE (2006) Impact of hemorrhage on trauma outcome: an overview of epidemiology, clinical presentations, and therapeutic considerations. JTrauma 60:S3-S11

2. Morrison JJ, Stannard A, Rasmussen TE, Jansen JO, Tai NR, Midwinter MJ (2013) Injury pattern and mortality of noncompressible torso hemorrhage in UK combat casualties. J Trauma Acute Care Surg 75:263-S268

3. Kleber C, Giesecke MT, Tsokos M, Haas NP, Buschmann CT (2013) Trauma-related preventable deaths in Berlin 2010: need to change prehospital management strategies and trauma management education. World J Surg 37:1154-1161

4. Tien HC, Spencer F, Tremblay LN, Rizoli SB, Brenneman FD (2007) Preventable deaths from hemorrhage at a level I Canadian trauma center. JTrauma 62:142-146

5. Sanddal TL, Esposito TJ, Whitney JR et al (2011) Analysis of preventable trauma deaths and opportunities for trauma care improvement in Utah. J Trauma 70:970-977

6. Morrison JJ, Rasmussen TE (2012) Noncompressible torso hemorrhage: a review with contemporary definitions and management strategies. Surg Clin North Am 92:843-858

7. Kisat M, Morrison JJ, Hashmi ZG, Efron DT, Rasmussen TE, Haider AH (2013) Epidemiologyand outcomes of non-compressible torso hemorrhage. JSurg Res 184:414-421

8. Edwards WS, SalterPP, Carnaggio VA (1953) Intraluminal aortic occlusion as a possible mechanism for controlling massive intra-abdominal hemorrhage. Surg Forum 4:496-499

9. Hughes CW (1954) Use of an intra-aortic balloon catheter tamponade for controlling intraabdominal hemorrhage in man. Surgery 36:65-68

10. Ng AC, Ochsner EC (1977) Use of Fogarty catheter tamponade for ruptured abdominal aortic aneurysms. Am J Roentgenol 128:31-33

11. Howard ER, Young AE (1971) Control of aortic haemorrhage by balloon catheter. Br Med J 3:161

12. Kulla M, Popp E, Knapp J (2019) Resuscitative endovascular balloon occlusion of the aorta: an option for noncompressible torso hemorrhage? Curr Opin Anaesthesiol 32:213-226

13. Knapp J, Bernhard M, Haltmeier T et al (2018) Resuscitative endovascular balloon occlusion of the aorta. Option for incompressible trunk bleeding? Anaesthesist 67:218-229

14. Hilbert-Carius $P$, Hauer $T$, Josse $F$ et al (2020) REBOA-resuscitative endovascular balloon occlusion of the aorta. Notarzt 36:33-45

15. DuBose JJ, Scalea TM, Brenner M et al (2015) The AAST prospective Aortic Occlusion for Resuscitation in Trauma and Acute Care Surgery (AORTA) registry: data on contemporary utilization and outcomes of aortic occlusion and resuscitative balloon occlusion of the aorta (REBOA). J Trauma Acute Care Surg 81:409-419

16. S3-Leitlinie Polytrauma/SchwerverletztenBehandlung https://www.awmf.org/uploads/ tx_szleitlinien/012-019l_S3_Polytrauma_ Schwerverletzten-Behandlung_2017-08.pdf. Zugegriffen:30.Dez. 2021

17. S3-Leitlinie zu Screening, Diagnostik und Therapie des Bauchaortenaneurysmas https://www. awmf.org/uploads/tx_szleitlinien/004-014l_ S3_Bauchaortenaneurysma_2018-08.pdf.Zugegriffen:30.Dez. 2021

18. Spahn DR, Bouillon B, Cerny V et al (2019) The European guideline on management of major 
bleeding and coagulopathy following trauma:fifth edition. Crit Care 23:98

19. Borger van der Burg BLS, Kessel B, DuBose JJ, Hörer TM, Hoencamp R (2019) Consensus on resuscitative endovascular balloon occlusion of the Aorta: a first consensus paper using a Delphi method. Injury 50:1186-1191

20. Brenner M, Bulger EM, Perina DG et al (2018) Joint statement from the American College of Surgeons Committee on Trauma (ACS COT) and the American College of Emergency Physicians (ACEP) regarding the clinical use of Resuscitative Endovascular Balloon Occlusion of the Aorta (REBOA). Trauma Surg Acute Care Open 3:1-3

21. Evidence report: Resuscitative endovascular balloon occlusion of the aorta in NSW trauma. https://aci.health.nsw.gov.au/_data/ assets/pdf_file/0020/651332/ACI-ResuscitativeEndovascular-Balloon-Occlusion-of-the-Aortain-NSW-Trauma-Evidence.pdf. Zugegriffen: 30. Dez. 2021

22. Bulger EM, Perina DG, Quasim Z et al (2019) Clinical use of resuscitative endovascular balloon occlusion of the aorta (REBOA) in civilian trauma systems in the USA, 2019: a joint statement from the American College of Surgeons Committee on Trauma, the American College of Emergency Physicians, the National Association of Emergency Medical Services Physicians and the National Association of Emergency Medical Technicians. Trauma Surg Acute Care Open 4:e376

23. Castellini G, Gianola S, Biffi A et al (2021) Resuscitative endovascular balloon occlusion of the aorta (REBOA) in patients with major trauma and uncontrolled haemorrhagic shock: a systematic review with meta-analysis. World $J$ Emerg Surg 16:41

24. Kulla M, Engelhardt M, Holsträter T et al (2018) Do we need REBOA as an adjunct to ER thoracotomy in German trauma centres? A secondary data analysis from the TraumaRegister $D G U^{\circledR}$. Anasth Intensivmed 59:562-573

25. Joseph B, Zeeshan M, Sakran JV et al (2019) Nationwide analysis of resuscitative endovascular balloon occlusion of the aorta in civilian trauma. JAMA Surg 154:500-508

26. Aoki R, Kobayashi Y, Nawata $S$ et al (2021) Computed tomography imaging of resuscitative endovascular balloon occlusion of the aorta (REBOA): pearls and pitfalls. Jpn J Radiol. https:// doi.org/10.1007/s11604-021-01166-w

27. Levis A, Egli N, Jenni H, Hautz WE, Daley Jl, Haenngi M (2021) Use of a disposable vascular pressure device to guide balloon inflation of resuscitative endovascular balloon occlusion of the aorta - a bench study. Sci Rep 11:24055

28. Michael M, Bax S, Finke M et al (2020) Aktuelle IstAnalyse zur Situation des nichttraumatologischen Schockraummanagementsin Deutschland. Notfall Rettungsmed. https://doi.org/10.1007/s10049020-00827-z

\section{Resuscitative endovascular balloon occlusion of the aorta in severely injured patients in the emergency trauma room: a case series}

Hemorrhage is the cause of death in $30-40 \%$ of severely injured patients due to trauma and the most frequent avoidable cause of death. In civilian emergency medical services, the majority of life-threatening hemorrhages are found in incompressible body regions (e.g. abdomen and pelvis). Resuscitative endovascular balloon occlusion of the aorta (REBOA) has therefore been discussed in recent years as a lifesaving procedure for temporary bleeding control in multiple trauma patients. Since August 2020 REBOA is implented in the treatment of seriously injured patients in the emergency department of the University Hospital of Bern. In this case series we report on our experiences in all seven patients in whom we performed this procedure during the first year.

\section{Keywords}

REBOA $\cdot$ Endovascular procedures $\cdot$ Multiple trauma $\cdot$ Resuscitation $\cdot$ Shock
MED UPDATE SEMINARE 2022

\section{Anästhesie Update 2022}

13. Anästhesie-Intensiv-Update-Seminar

11.-12. November 2022

Mainz und Livestream

Wiss. Leitung:

Prof. Dr. Grietje Beck, Wiesbaden

Prof. Dr. Winfried Meißner, Jena

Prof. Dr. Dr. Kai Zacharowski, Frankfurt

Unter der Schirmherrschaft der BDA

www.anaesthesie-update.com

Auskunft für alle Update-Seminare: med update $\mathrm{GmbH}$

www.med-update.com

Tel.: 0611 - 736580

info@med-update.com 